und als Zusatz durch das Feld:

$$
L^{\prime}=\mathfrak{\Re} \cdot \frac{\mathrm{d} \mathfrak{p}}{\mathrm{d} t} \tau \frac{1}{m} .
$$

Mit $\Re=\mathrm{d} \mathfrak{p} / \mathrm{d} t=-e \mathfrak{r}$ und der Ersetzung

$$
\mathfrak{R} \cdot \mathfrak{R} \longrightarrow \mathfrak{R} \cdot \mathfrak{T}
$$

ergibt sich daraus, wenn man noch über die Teilflächen summiert:

$$
L^{\prime}=e^{2} \sum \mathfrak{V} \mathfrak{F} \cdot(\mathfrak{T} \cdot \Phi)
$$

Die geforderte Gleichheit der Leistungsaufnahme liefert (2.7). Man bekommt (2.7) natürlich auch durch Einsetzen von (6.11) und (6.12) in (1.11) bzw. (1.13).

Meinem verehrten Lehrer, Herrn Professor Dr. W $\mathrm{WL}_{\mathrm{AL}}$ TER Franz, danke ich für die Anregung zu dieser Arbeit und für fördernde Diskussionen.

\title{
Uber das Verhalten von n-Ge-Einkristallen im Temperaturgebiet des flüssigen Heliums.
}

\author{
Von Günter Finke und Günter Lautz \\ Aus dem Institut für technische Physik der Technischen Hochschule Braunschweig \\ (Z. Naturforschg. 14 a, 62-74 [1959]; eingegangen am 10. September 1958)
}

\begin{abstract}
Nach theoretischen Betrachtungen von Conwell und Motr soll der Übergang vom normalen Leitungsmechanismus eines Halbleiters zur Störbandleitung im Gebiet tiefster Temperaturen durch eine Kompensation der Störstellenarten des Kristalls begünstigt werden. In der vorliegenden Arbeit wird durch Messungen der Temperaturabhängigkeit des elektrischen Widerstandes und des Hall-Effektes für kleine elektrische Felder an n-Ge-Einkristallen unterschiedlicher Störstellenstruktur diese theoretische Aussage auch experimentell belegt.

Die oberhalb einer bestimmten elektrischen Feldstärke $U_{\mathrm{D}}$ von einigen Volt/cm für Meßtemperaturen unter $10^{\circ} \mathrm{K}$ beobachtete reversible Abnahme des elektrischen Widerstandes und der HallKonstanten in Abhängigkeit vom angelegten Feld kann bei normalem Leitungsmechanismus mit einer Ladungsträgervervielfachung durch Stoßionisation neutraler Störstellen gedeutet werden. Diese Erklärung wird insbesondere durch die Abnahme der „Durchschlagsfeldstärke“ $U_{\mathrm{D}}$ mit zunehmender Beweglichkeit der Ladungsträger und durch die Zunahme von $U_{D}$ in einem äußeren Magnetfeld gestützt. Quantitativ können durch die Behinderung der Stoßionisation in einem Magnetfeld außergewöhnlich hohe Widerstandsänderungen von $10000 \%$ bei $300 \mathrm{Gauß}$ für elektrische Feldstärken $U \approx U_{\mathrm{D}}$ beobachtet werden. Im Bereich überwiegender Störbandleitung wird zur Deutung der Feldstärkeabhängigkeiten des Widerstandes und des Hall-Effektes nach Sсноттку ein neuer Mechanismus der "Ionisierung“" von Elektronen im Störband benutzt.
\end{abstract}

\section{Einführung und Problemstellung}

Experimentelle Untersuchungen an Halbleitern bei hohen Temperaturen führen in den meisten Fällen zu recht übersichtlichen Ergebnissen, da die Stoffeigenschaften im wesentlichen durch das Grundgitter bestimmt sind. Bei tiefen Temperaturen hingegen gewinnen die verschiedenen Störstellen entscheidenden Einfluß, so daß die Deutung derartiger Versuche erheblich erschwert wird. Es ist daher nicht verwunderlich, wenn es selbst bei den gut bekannten Elementhalbleitern $\mathrm{Ge}$ und $\mathrm{Si}$ im Tieftemperaturgebiet Effekte gibt, die wohl qualitativ, aber noch nicht quantitativ erklärt werden können. Das trifft insbesondere für diejenigen Meßresultate zu, die nicht den üblichen Erwartungen über das Verhalten eines Störstellenhalbleiters entsprechen und in diesem Sinne Anomalien sind. $\mathrm{Zu}$ diesen anomalen Effekten gehören z. B. die unterhalb
$10{ }^{\circ} \mathrm{K}$ beobachtete Störbandleitung und die schon in kleinen elektrischen Feldern von nur wenigen Volt $/ \mathrm{cm}$ ermittelte Ladungsträgervervielfachung durch Stoßionisation, die ebenfalls erst im Heliumtemperaturgebiet besonders hervortritt.

Während ein idealer Störstellenhalbleiter zu tiefen Temperaturen hin eine exponentielle Abnahme der elektrischen Leitfähigkeit und eine äquivalente Zunahme der HaLL-Konstanten zeigen sollte, konnten schon Busch und LabHart ${ }^{1}$ am $\mathrm{SiC}$ und etwas später Hung und Gliesman ${ }^{2,3}$ am Ge feststellen, daß unterhalb einer von Probe zu Probe verschiedenen Temperatur die Aktivierungsenergie der Trägererzeugung erheblich abnimmt und die Hall-Konstante ein Maximum durchläuft. Systematische Un-

1 G. Busch u. H. Labhart, Helv. Phys. Acta 19, 463 [1946].

2 C. S. Hung, Phys. Rev. 79, 726 [1950].

3 C. S. Hung u. J. R. Gliesman, Phys. Rev. 79, 727 [1950]. 
tersuchungen von Hung und GLIesman ${ }^{4}$ und FritzSCHE und Lark-HoRovitz ${ }^{5,6}$ ergaben qualitativ, daß der Effekt bei um so höheren Temperaturen einsetzt, je größer die Störstellendichte des Kristalls ist.

Grob anschaulich können diese Experimente durch einen direkten Elektronenübergang von Störstelle zu Störstelle als Störbandleitung plausibel gemacht werden. Doch ist eine befriedigende theoretische Klärung bisher nicht möglich, da die Störstellenkonzentrationen im Versuchsmaterial viel niedriger liegen, als theoretisch für eine derartige Wechselwirkung zu fordern ist. Nach den ersten Rechnungen mit einer regelmäßigen, gitterartigen Störstellenverteilung im Wirtskristall ${ }^{3,7,8}$ zeigen die Arbeiten von James und Ginzbarg ${ }^{9}$ bzw. Aigrain ${ }^{10}$, daß auch eine statistische Verteilung diese grundsätzliche Schwierigkeit nicht zu beheben vermag. Auch die Berücksichtigung der Wechselwirkungen zwischen den angeregten Zuständen benachbarter Störstellen blieb ohne quantitativen Erfolg ${ }^{11}$. Nach Conwell ${ }^{12}$ und Moтr ${ }^{13}$ ist das Ausmaß der p-n-Kompensation für das Zustandekommen der Störbandleitung von großer Bedeutung. Nur bei relativ stark p-n-kompensierten Proben kann gewährleistet sein, daß z. B. im Donatorenband eine hinreichende Anzahl freier Plätze für den Elektronentransport verfügbar ist. Aus diesem Grunde werden in der vorliegenden Arbeit Tieftemperaturmessungen an einer eigenleitenden Ge-Probe mit einem Zimmertemperatur-Widerstand von $40 \mathrm{Ohm} \cdot \mathrm{cm}$, an einer mit $\mathrm{Sb}$ bis auf $5 \mathrm{Ohm} \cdot \mathrm{cm}$ dotierten Probe und schließlich an einem weiteren Kristall vorgenommen, der erst mit der gleichen Sb-Menge und zusätzlich mit In wieder auf den Wert von $40 \mathrm{Ohm} \cdot \mathrm{cm}$ gegendotiert worden ist. Ein Vergleich der Störbandleitung dieser Proben sollte den Einfluß der p-n-Kompensation deutlich machen.

${ }^{4}$ C. S. Hung u. J. R. Gliesman, Phys. Rev. 96, 1226 [1954].

5 H. Fritzsche u. K. Lark-Horovitz, Physica 20, 834 [1954].

${ }^{6}$ H. Fritzsche, Phys. Rev. 99, 406 [1955].

7 W. Baltensperger, Phil. Mag. (7) 44, 1355 [1953].

8 F. Stern u. R. M. Talley, Phys. Rev. 100, 1638 [1955].

9 H. M. James u. A. S. Ginzbarg, J. Phys. Chem. 57, 840 [1953].

10 P. Aigrain, Physica 20, 978 [1954].

11 C. Erginsoy, Phys. Rev. 80, 1104 [1950].

12 E. M. Conwell, Phys. Rev. 103, 51 [1956].

13 N. F. Мотт, Canad. J. Phys. 34, 1356 [1956].

14 I. Esterman, A. Foner u. I. E. Zimmerman, Phys. Rev. 75, 1631 [1949].

15 A. N. Gerritsen, Physica 15, 427 [1949].

16 D. F. K. Du PrÉ, Phys. Rev. 77, 152 u. 571 [1950].

17 E. M. Conwell, Phys. Rev. 90, 769 [1953]; 94, 1068 [1954].
Die bei tiefen Temperaturen ermittelte Stoßionisation in Ge-Kristallen ist mit unseren bisherigen Kenntnissen ebenfalls nur qualitativ verständlich. Hier fehlen aber auch noch eingehende und systematische experimentelle Untersuchungen. Der Effekt als solcher wurde 1949 von Esterman, Foner und Zimmerman $^{14}$ und Gerritsen ${ }^{15}$ gefunden. Die Abweichungen der Strom-Spannungs-Charakteristik vom OHMschen Verhalten wurden von Du PrÉ ${ }^{16}$ mit lokalen p-n-Übergängen und von ConwelL ${ }^{17}$ irrtümlich mit einer Feldstärkeabhängigkeit der Trägerbeweglichkeit interpretiert, bis Sclar, Burstein, Turner und Davisson ${ }^{18}$ die Meßergebnisse durch reversible „Durchschlagsvorgänge“ infolge einer Stoßionisation neutraler Störstellen zu deuten versuchten. Durch die in der Folgezeit erschienenen Arbeiten verschiedener Autoren ${ }^{19-27}$ wird diese Auffassung qualitativ erhärtet. Im wesentlichen ist dabei die Widerstandsabnahme mit steigendem elektrischem Feld, die Abhängigkeit der „Durchschlagsfeldstärke“ von der Temperatur und der Trägerbeweglichkeit, sowie das zeitliche An- und Abklingen der Lawinenbildung erfaßt worden. In der vorliegenden Arbeit sollen neue quantitative Ergebnisse über den Zusammenhang zwischen dem Einsetzen der Stoßionisation und der Probentemperatur, der Störstellenstruktur der Proben und insbesondere über die magnetische Beeinflussung der Ladungsträgervervielfachung mitgeteilt werden. Diese Erweiterung der experimentellen Fakten ist wünschenswert, um für die theoretische Beschreibung des Leitungsmechanismus schneller Elektronen neue Anhaltspunkte zu ermitteln.

\section{Experimentelles}

Für die Messungen im Temperaturbereich von $10^{\circ} \mathrm{K}$ bis $300{ }^{\circ} \mathrm{K}$ diente ein wechselweise mit flüssigem Stickstoff oder mit flüssigem Wasserstoff betreibbarer Thermo-

18 N. Sclar, E. Burstein, W. Turner u. I. W. Davisson, Phys. Rev. 91, 215 [1953].

19 N. Sclar, E. Burstein u. I. W. Davisson, Phys. Rev. 92, 858 [1953].

20 E. J. Ryder, I. M. Ross u. D. A. Kleinman, Phys. Rev. 95, 1342 [1954].

21 S. H. Koenig u. G. R. Gunther-Mohr, Phys. Rev. 98, 228 [1955].

22 F.J.Darnell u. S.A. Friedberg, Phys. Rev. 98, 1178 u. 1860 [1955].

23 G. Finke u. G. Lautz, Z. Naturforschg. 12 a, 223 [1957].

24 G. Finke, Dissertation, Braunschweig 1957.

25 A. I. Abaulina-Zavaritskaia, Zh. eksper. teor. Fiz. 30, 1158 [1956] ; J. Exp. Theor. Phys., USSR 3, 984 [1957].

26 N. Sclar u. E. Burstein, J. Phys. Chem. Solids 2, 1 [1957].

27 S. H. Koenig u. G. R. Gunther-Mohr, J. Phys. Chem. Solids 2, 268 [1957]. 
stat nach Abb. 1 28. Der Wärmeaustausch zwischen der im Inneren befindlichen und von einem Blei-Thermometer umgebenen Probe und dem äußeren Kältebad war durch den He-Gasdruck im Dewarmantel des Metallgefäßes bequem den experimentellen Erfordernissen anpaßbar. Die Heizung gab zudem eine weitere Temperaturregelmöglichkeit. Für Temperaturen unter $20^{\circ} \mathrm{K}$ wurde der Innenteil mit flüssigem Wasserstoff gefüllt und abgepumpt, während im Außenraum flüssiger Wasserstoff die Wärmeeinstrahlung hinreichend begrenzte. Zwischen $20^{\circ} \mathrm{K}$ und $80^{\circ} \mathrm{K}$ befand sich die Probe im

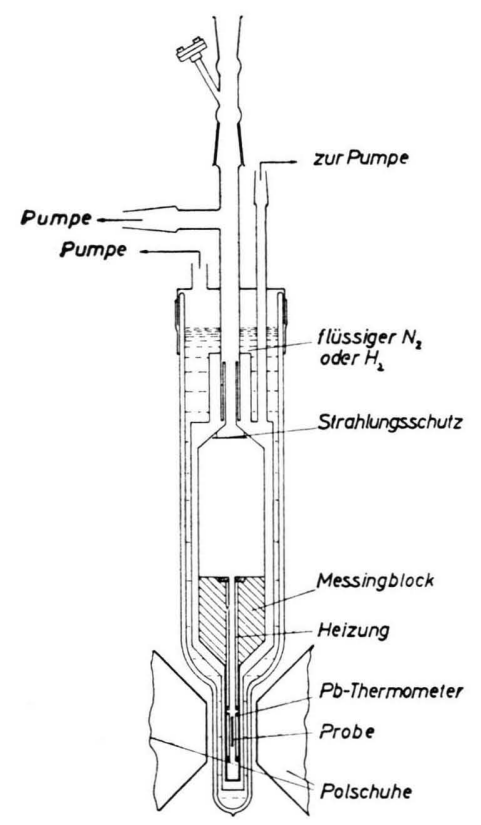

Abb. 1. Thermostat für Messungen zwischen $10^{\circ} \mathrm{K}$ und $300^{\circ} \mathrm{K}$.

Innenraum in einer gasförmigen Wasserstoffatmosphäre. Das Temperaturgleichgewicht zwischen Gas, Thermometer und Probe konnte durch die verdickten Metallteile und eine möglichst enge Ausführung aus dem unteren Teil gegenüber üblichen Anordnungen verbessert werden. Die zeitliche Temperaturkonstanz war weitaus genügend, um einwandfreie Messungen an den Ge-Kristallen ausführen zu können.

Für das Temperaturgebiet unter $10^{\circ} \mathrm{K}$ benutzten wir einen He-Expansionsverflüssiger, dessen Schnittzeichnung in Abb. 2 wiedergegeben ist. Die Cu-Druckbombe wurde im Betrieb mit Wasserstoff auf $12{ }^{\circ} \mathrm{K}$ vorgekühlt und mit 170 atü $\mathrm{He}$ gefüllt. Von einer gesonderten Evakuierung des Zwischenmantels zwischen dem äußeren Wasserstoffbad und der inneren He-Bombe konnte abgesehen werden, da die Aktivkohle in Kontakt mit dem festen Wasserstoff enthaltenden Gefäßteil die Luftreste völlig absorbiert und damit die erforderliche thermische Isolierung der Druckbombe erreicht. Bei einer Expansionszeit von 15 Min. konnten etwa

28 W. Ruppel, Dissertation, Braunschweig 1955.
$80 \%$ des $130 \mathrm{~cm}^{3}$ großen Innenvolumens mit flüssigem He gefüllt werden. Diese Menge war ausreichend, um im Meßraum $\left(10 \mathrm{~cm}^{3}\right)$ bis zu 5 Stunden die Siedetemperatur des flüssigen $\mathrm{He}$ zu halten. Beim Abpumpen verringerte sich die zur Verfügung stehende Meßzeit auf ca. 90 Min.

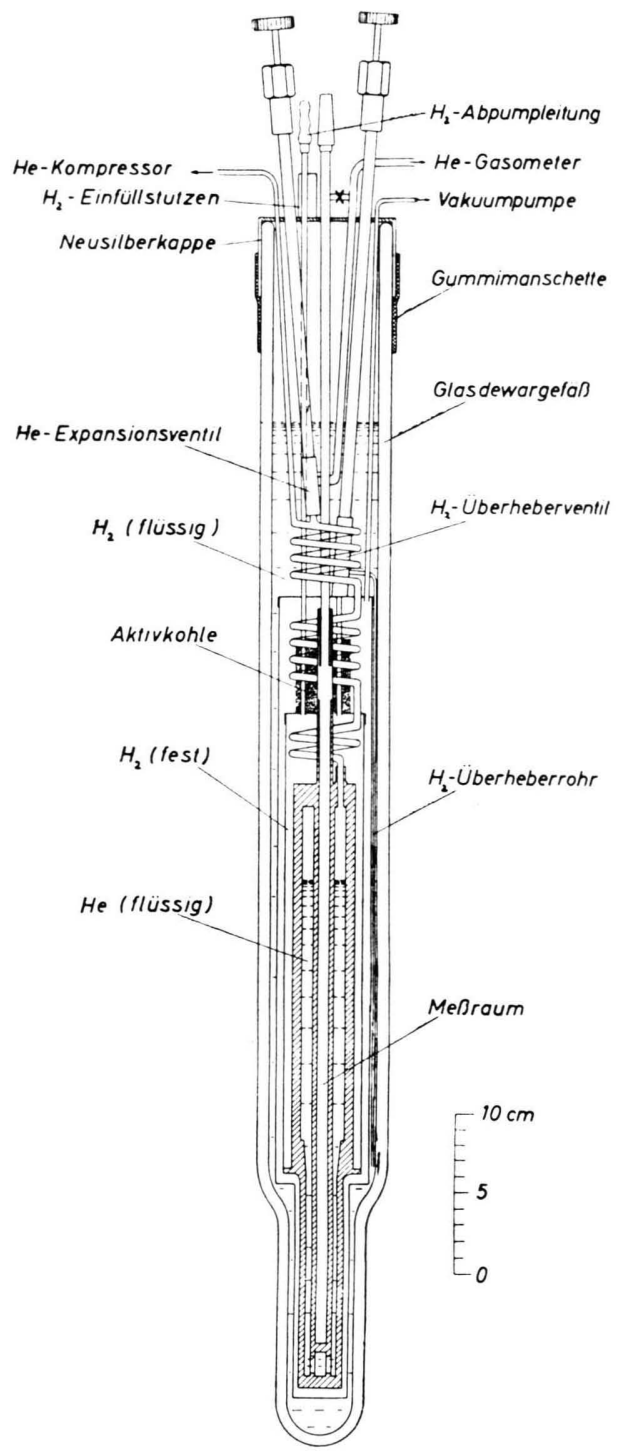

Abb. 2. Helium-Expansions-Verflüssiger für Messungen zwischen $4,2{ }^{\circ} \mathrm{K}$ und $11{ }^{\circ} \mathrm{K}$.

bei $1,95{ }^{\circ} \mathrm{K}$. Der Bedarf an flüssigem Wasserstoff beträgt für die erste Verflüssigung etwa 1,7 Liter. Wird die Verflüssigung unmittelbar nach dem Verdampfen des letzten $\mathrm{He}$ wiederholt, sind nurmehr 0,6 Liter flüssiger Wasserstoff für eine erneute $\mathrm{He}$-Verflüssigung notwendig. Die für die zweite Verflüssigung erforderliche Zeit zum Komprimieren, Abkühlen und Expandieren des He kann auf 30 Min. reduziert werden. 
Für die vorliegenden Messungen war es zudem von großem Vorteil, daß durch die Einstellung des Expansionsventils jede Temperatur zwischen $4,2^{\circ} \mathrm{K}$ und $11^{\circ} \mathrm{K}$ mit großer Genauigkeit über Stunden hinweg konstant zu erhalten war. In dieser Weise konnten Messungen oberhalb $4,2{ }^{\circ} \mathrm{K}$ unabhängig von Temperaturschwankungen oder -wanderungen ausgeführt werden.

Um ein leichtes und schnelles Auswechseln der Meßproben zu ermöglichen, ist die He-Druckbombe als Zylinderring ausgebildet worden. Der Meßling konnte so von oben her in das innere Rohr eingeführt werden, wobei der Wärmekontakt zur He-Druckbombe durch gasförmiges $\mathrm{He}$ von $10-50$ Torr hergestellt wurde. Zur definierten Orientierung des Kristalls im Magnetfeld wurde die Probe an einem Quarzstab befestigt und mittels einer Schliffverbindung auch während der Messung um die Längsachse gedreht.

Die Temperaturbestimmung erfolgte mit einem zuvor geeichten Kohle-Schicht-Widerstand, der auch bei mehrfachem Abkühlen und Wiedererwärmen eine streng reproduzierbare exponentielle Widerstands-TemperaturKennlinie aufwies. Unter $10{ }^{\circ} \mathrm{K}$ ist eine Genauigkeit von $5 / 1000^{\circ} \mathrm{K}$ leicht erreichbar. Die magnetische Widerstandsänderung dieses Kohle-Thermometers war so gering, daß bei Messungen im Magnetfeld keine Korrekturen an den Meßwerten anzubringen waren. Der Wärmekontakt zwischen Probe und Thermometer wurde durch ein beide Teile eng umschließendes Cu-Rohr verbessert.

Um einwandfreie und reproduzierbare Meßergebnisse zu erhalten, wurden zur Unterdrückung von Oberflächeneffekten ${ }^{6}$ die Ge-Kristalle geätzt, anschließend einige Minuten in heißem und kaltem destilliertem Wasser gespült und mit Filterpapier getrocknet. Sperrschichtfreie Elektroden konnten mit einer $\mathrm{Sn}-\mathrm{Pb}$-Legierung erzielt werden, der etwas $\mathrm{Sb}$ zugesetzt war.

Für die elektrischen Messungen benutzten wir bei Probenwiderständen unter $100 \mathrm{kOhm}$ einen fünfstufigen Dießelhorst-Kompensator, zwischen 100 und $1000 \mathrm{kOhm}$ einen Gleichstromverstärker nach $\mathrm{K}_{\text {NICK }}$ (Eingangswiderstand $>10^{10} \mathrm{Ohm}$ ) und oberhalb $1000 \mathrm{kOhm}$ ein Wulffsches Einfaden-Elektrometer. Die Bestimmung der Widerstände erfolgte durch Vergleich mit geeichten Normalwiderständen. Bei jeder Messung wurde zudem die Stromrichtung zur Kontrolle auf evtl. Sperrschichten umgepolt. Die getrennten Strom- und Potentialdrähte waren glasisoliert und auf Trolitul gehaltert. Die Isolationswiderstände lagen im allgemeinen über $10^{14}$ Ohm.

\section{Verhalten bei kleinen elektrischen Feldern $\left(U \ll U_{\mathrm{D}}\right)$}

Um zunächst unabhängig von einer durch elektrische Feldwirkung bedingten Ladungsträgervermehrung die Eigenschaften der verschiedenen Kristalle zu ermitteln, mußten die Versuche mit hinreichend kleinen elektrischen Feldstärken $U$ ausgeführt werden. Nach unseren früheren Untersuchun- gen $^{23}$ war es insbesondere im Temperaturgebiet zwischen $8{ }^{\circ} \mathrm{K}$ und $12{ }^{\circ} \mathrm{K}$ notwendig, $U<0,5 \mathrm{Volt} / \mathrm{cm}$ zu wählen. Außerhalb des genannten Bereichs liegen die Feldstärken $U_{\mathrm{D}}$ für das Einsetzen der Stoßionisation so hoch, daß bei den üblichen Gleichstromanordnungen mit 4-6-Volt-Batterien keine Beschränkun-

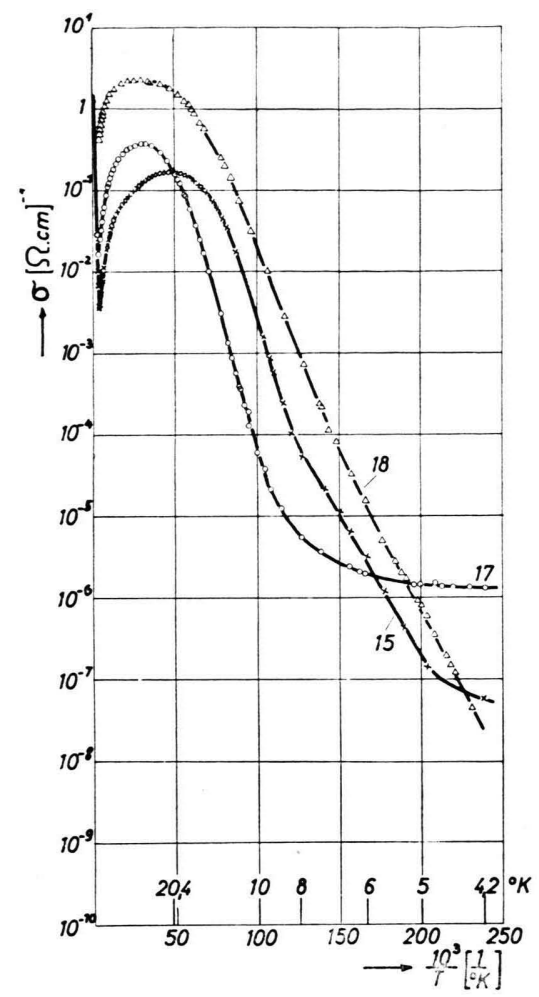

Abb. 3. Temperaturabhängigkeit der elektrischen Leitfähigkeit verschiedener n-Ge-Einkristalle zwischen $4,2^{\circ} \mathrm{K}$ und $300^{\circ} \mathrm{K}$.

gen erforderlich sind. Die Belastungsströme bleiben unter diesen Bedingungen so klein, daß keine schädlichen Erwärmungen auftreten. Zur Sicherheit wurde die Gültigkeit des Oнмschen Gesetzes in jedem Einzelfall überprüft.

In den Abb. 3 - 5 sind die Temperaturabhängigkeiten der elektrischen Leitfähigkeit, der HaLL-Konstanten und des Produktes $\left|A_{\mathrm{H}}\right| \sigma$ dargestellt worden. Bei den Hall-Effekt-Bestimmungen hatte die magnetische Feldstärke innerhalb der Meßgenauigkeit keinen Einfluß auf die Größe $\operatorname{der} A_{\mathrm{H}}$-Werte. Die geometrischen Bedingungen (Verhältnis Länge zu Breite der Probe, Potentialsondenabstand vom Rand) waren stets so gewählt, daß keine Verfälschungen der Resultate auftreten konnten. Bevor wir auf eine Auswertung und Diskussion eingehen, sollen noch einige Bemerkungen über die Kontroll- 
messungen eingefügt werden, die sicher zeigen, daß die beobachteten Ergebnisse echte Volumeneffekte sind.

Unterschiedliche Ätzmethoden und eine Veränderung der geometrischen Dimensionen in bezug auf das Verhältnis Volumen/Oberfläche hatten keinerlei Einfluß auf das jeweilige Meßresultat. Zudem wurde festgestellt, daß ein transversales elektrisches Feld

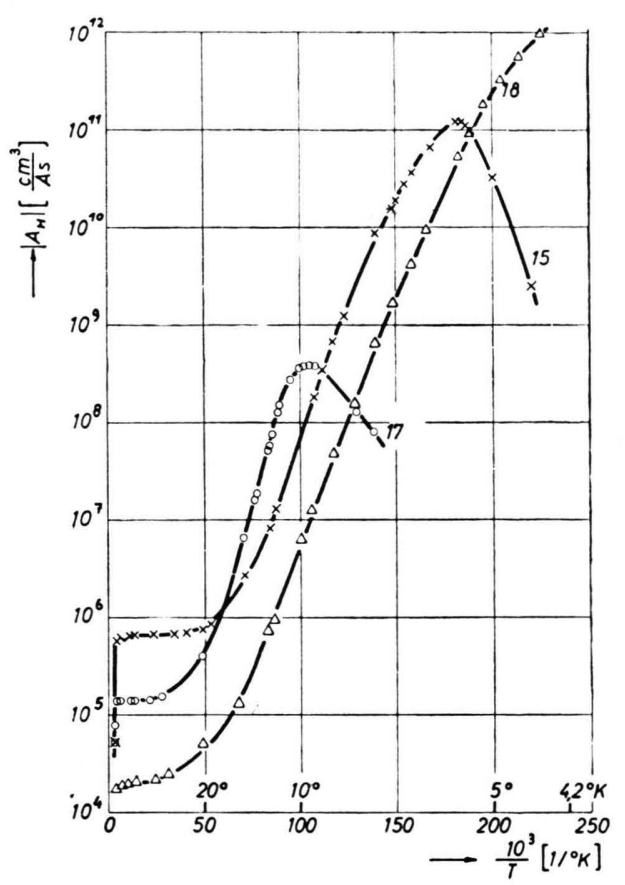

Abb. 4. Temperaturabhängigkeit der HALL-Konstanten verschiedener n-Ge-Einkristalle zwischen $4,2{ }^{\circ} \mathrm{K}$ und $300{ }^{\circ} \mathrm{K}$.

von $2200 \mathrm{Volt} / \mathrm{cm}$ bei beliebiger Polung keine Veränderungen der Probeneigenschaften hervorrief. Wären Oberflächenrandschichten entscheidend an den beobachteten Effekten beteiligt, hätten sich unter dieser Feldeinwirkung Abweichungen $>1 \%$ von den ohne Feld bestimmten Transportgrößen ergeben müssen. Eine Wiederholung der Versuchsreihen mit neu angelöteten Kontakten erbrachte die gleichen Resultate. Die zeitlich irreversiblen Änderungen blieben sicher $<3 \%$.

Um näheren Aufschluß über die Störstellenart und den Störstellengehalt der Einzelproben zu bekommen, wollen wir die $A_{\mathrm{H}}(T)$-Abhängigkeit im Gebiet der reinen Störstellenhalbleitung quantitativ

29 W. Shoскцеy, Electrons and Holes in Semiconductors, Van Nostrand, New York 1950, S. 472.

30 C. Herring. Bell Syst. Tech. J. 34. 237 [1955].

31 T. H. Geballe u. F. J. Morin, Phys. Rev. 95, 1085 [1954]. auswerten. Da unter $20^{\circ} \mathrm{K}$ die Trägerdichte $n$ sehr klein gegen die Dichte $N_{\mathrm{D}}-N_{\mathrm{A}}$ im Gebiet vollständiger Ionisation und, wie sich zeigen wird, auch $n$ noch klein gegen die Akzeptorkonzentration $N_{\mathrm{A}}$ bleibt, können wir nach Shockley ${ }^{29}$ die Beziehung

$$
n=\frac{N_{\mathrm{D}}-N_{\mathrm{A}}}{N_{\mathrm{A}}} 2 g_{\mathrm{D}}\left(\frac{2 \pi m_{\mathrm{d}} k T}{h^{2}}\right)^{3 / 2} e^{-\Delta E_{\mathrm{D}} / k T}
$$

benutzen, wobei $g_{D}$ das Verhältnis der statistischen Gewichte der freien und der gebundenen Elektronenzustände am Donator, $\Delta E_{\mathrm{D}}$ die Aktivierungsenergie der Donatoren und $m_{\mathrm{d}}$ die durch

$$
\left.\left.m_{\mathrm{d}}=N_{\mathrm{V}^{2 / 3}}{ }^{2 / 3} m_{\perp}{ }^{2} m_{\|}\right)^{1 / 3} \quad \text { (s. Anm. }{ }^{30}\right)
$$

$\left(N \mathbf{v}=\right.$ Zahl der Ellipsoide im f-Raum, $m_{\perp}=$ transversale, $m \|=$ longitudinale Masse) gegebene „density-of-states"-Masse der Elektronen bedeuten. Der Zusammenhang mit $A_{\mathrm{H}}$ wird durch

$$
A_{\mathrm{H}}=r / n e
$$

bestimmt, wobei $r$ je nach der Art des Streumechanismus Werte zwischen 1 und 2 annimmt. Aus der Temperaturabhängigkeit des $\left|A_{\mathrm{H}}\right| \sigma$ ergibt sich, daß sich die Proben 15 und 18 bis zu $10^{\circ} \mathrm{K}$ noch im Bereich überwiegender Gitterstreuung befinden, während bei der Probe 17 bei $20^{\circ} \mathrm{K}$ das Maximum der HaLL-Beweglichkeit schon überschritten ist und hier die Streuung an ionisierten Zentren hervortritt. Wir wollen daher bei den Kristallen 15 und $18 r=3 \pi / 8$ und bei $17 r=315 \pi / 512$ näherungsweise einsetzen. In dieser Weise wird auch das Überschneiden der $A_{\mathrm{H}}(T)$-Kurven der Proben 15 und 17 zwanglos gegeutet. Die nunmehr aus dem Anstieg ermittelten $\Delta E_{\mathrm{D}}$-Werte sind in die Tab. 1 eingetragen worden. $\mathrm{Sb}$ ist in allen Fällen als wirksamer Donator anzusehen. Der geringfügig erhöhte Wert bei Probe 17 entspricht ähnlichen Beobachtungen anderer Autoren ${ }^{31}$ bei p-n-kompensierten Elementhalbleitern. Die im Gebiet vollständiger Ionisation unmittelbar ablesbaren Konzentrationen $N_{\mathrm{D}}-N_{\Lambda}$ sind bei allen Kristallen mit $r=3 \pi / 8$ ermittelt worden. Zur Berechnung des $N_{\mathrm{A}}$ sollen $g_{\mathrm{D}}=1 / 2, N_{\mathrm{V}}=4$ für n-Ge und $m_{\perp}$ und $m_{\|}$mit ihren bekannten Zyklotronresonanzwerten eingesetzt werden ${ }^{32}$. Mit den so aus Gl. (1) bestimmten $N_{\mathrm{A}}$ sind dann die ebenfalls in der Tabelle enthaltenen $N_{\text {D }}$ gefunden worden. Die Größe $\left(N_{\mathrm{D}}-N_{\mathrm{A}}\right) / N_{\mathrm{D}}$ kann als Maß der p-n-Kom-

32 V. A. Johnson u. K. Lark-Horovitz, Progress in Low Temperature Physics (C. J. Gorter), North Holland Publ. Co. Amsterdam 1957, S. 187. 


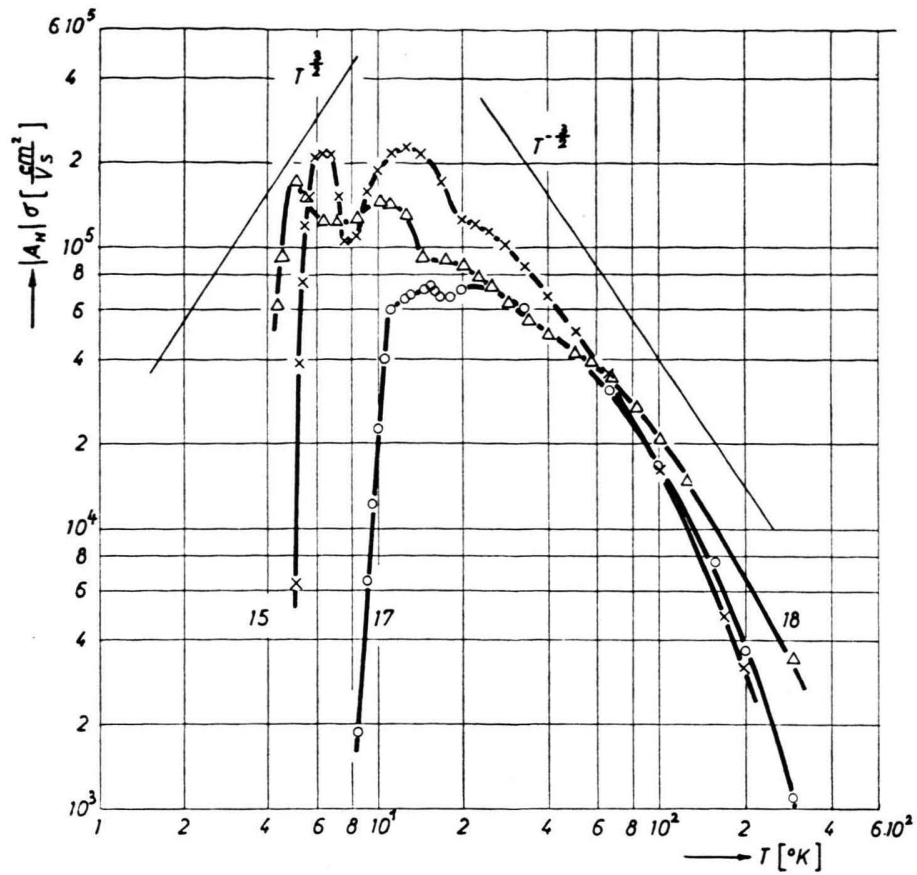

Abb. 5. Temperaturabhängigkeit der Größe $\left|A_{\mathrm{H}}\right| \sigma$ verschiedener n-Ge-Einkristalle zwischen $4,2{ }^{\circ} \mathrm{K}$ und $300{ }^{\circ} \mathrm{K}$.

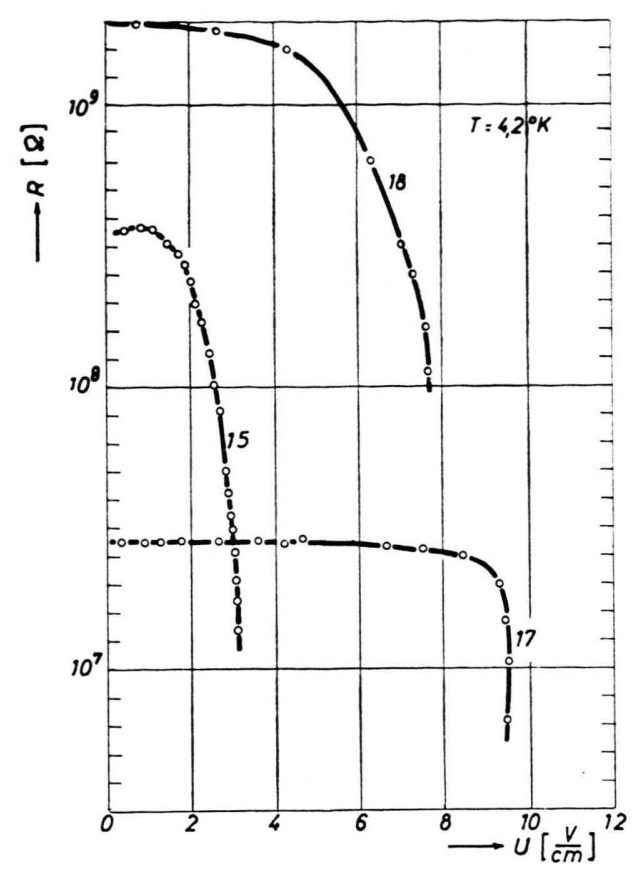

Abb. 6. Feldstärkeabhängigkeit des Widerstandes verschiedener $\mathrm{n}$-Ge-Einkristalle bei $4,2^{\circ} \mathrm{K}$. pensation angesehen werden. Sie wird 0 bei vollständiger Störstellenkompensation und 1 bei einem akzeptorfreien Überschußhalbleiter. Die erwähnten $\left(N_{\mathrm{D}}-N_{\mathrm{A}}\right) / N_{\mathrm{D}}$-Werte zeigen deutlich, daß die Probe 17 extrem stark gegendotiert ist, aber auch die eigenleitenden Kristalle noch als stark p-n-kompensiert anzusprechen sind.

Betrachten wir nun das Auftreten der Störbandleitung im Zusammenhang mit dieser Aussage, so geht aus unseren Messungen hervor, daß eine p-nKompensation günstigere Bedingungen für die Störbandleitung bietet, wie auch theoretisch zu fordern ist. Nur in diesem Fall hat das von Donator zu Donator weitergereichte Elektron eine Chance, in seiner unmittelbaren Nachbarschaft auch bei Konzentrationen unter $10^{17} \mathrm{~cm}^{-3}$ noch einen ionisierten Donator anzutreffen, zu dem ein Platzwechsel möglich ist. Extrem deutlich wird das bei einem Ver- gleich der Proben 15 und 18. Trotz höherer Störstellenkonzentration geht die Probe 18 erst bei tieferen Temperaturen zur Störbandleitung über als Probe 15. Hier stellen sich offenbar der Bewegung der Elektronen viele besetzte Donatoren hemmend in den Weg, während beim Kristall 15 praktisch jeder Donator von nichtbesetzten Donatoren umgeben ist. $\mathrm{Da} \beta$ die noch stärker p-n-kompensierte Probe 17 schon bei $10^{\circ} \mathrm{K}$ in die Störbandleitung einmündet, hängt u. a. auch noch mit dem höheren $N_{\mathrm{D}}+N_{\mathrm{A}}$ zusammen.

Qualitativ können diese Verhältnisse modellmäßig so dargestellt werden, daß die Beweglichkeit im Störband in extremem Maße von dem Besetzungsgrad abhängig ist. Obgleich z. B. bei der Probe 18 im Störband mehr Ladungsträger verfügbar sind und daher das Band selbst auch einen größeren Energiebereich überdeckt, wird die Bewegungsmög-

\begin{tabular}{|c|c|c|c|c|c|c|c|}
\hline $\begin{array}{l}\text { Probe } \\
\text { Nr. }\end{array}$ & $\begin{array}{c}\varrho \text { bei } \\
300^{\circ} \mathrm{K} \\
\mathrm{Ohm} \cdot \mathrm{cm}\end{array}$ & $\begin{array}{l}\text { Dotie- } \\
\text { rung }\end{array}$ & $\begin{array}{c}\Delta E_{\mathrm{D}} \\
\mathrm{eVolt}\end{array}$ & $\begin{array}{c}\left(N_{\mathrm{D}}-N_{\mathrm{A}}\right) \\
\mathrm{cm}^{-3}\end{array}$ & $\underset{\mathrm{cm}^{-3}}{N_{\mathrm{A}}}$ & $\underset{\mathrm{cm}^{-3}}{N_{\mathrm{D}}}$ & $\left(N_{\mathrm{D}}-N_{\mathrm{A}}\right) / N_{\mathrm{D}}$ \\
\hline $\begin{array}{l}15(14) \\
17 \\
18\end{array}$ & $\begin{array}{r}40 \\
40 \\
5\end{array}$ & $\begin{array}{c}- \\
\mathrm{Sb}+\mathrm{In} \\
\mathrm{Sb}\end{array}$ & $\begin{array}{l}0,0096 \\
0,0100 \\
0,0093\end{array}$ & $\begin{array}{l}1,1 \cdot 10^{13} \\
5,4 \cdot 10^{13} \\
3,5 \cdot 10^{14}\end{array}$ & $\begin{array}{l}6,3 \cdot 10^{13} \\
9,8 \cdot 10^{14} \\
2,1 \cdot 10^{14}\end{array}$ & $\begin{array}{r}7,4 \cdot 10^{13} \\
1,04 \cdot 10^{15} \\
5,6 \cdot 10^{14}\end{array}$ & $\begin{array}{l}0,15 \\
0,052 \\
0,63\end{array}$ \\
\hline
\end{tabular}

Tab. 1 . 
lichkeit im Mittel durch die höhere Bandauffüllung beim Kristall 18 ungleich stärker vermindert. Es bleibt jedoch hier wie auch bei älteren Messungen anderer Autoren unklar, warum bei einer über 50proz. Bandbesetzung keine Löcherleitung mit einer entsprechenden Vorzeichenumkehr des HALL-Effektes auftritt ${ }^{2,4,5,6}$.

Die Maxima der $A_{\mathrm{H}}(T)$-Kurven ermöglichen außerdem eine ungefähre Abschätzung der mittleren Beweglichkeit im Störband. Im allgemeinen Fall einer Zweibänderleitung gilt

$$
A_{\mathrm{HI}}=\begin{array}{cc}
1 & n_{1} \mu_{\mathrm{H} 1} \mu_{1}+n_{2} \mu_{\mathrm{H} 2} \mu_{2} \\
e & \left(n_{1} \mu_{1}+n_{2} \mu_{2}\right)^{2}
\end{array}
$$

bzw.

$$
\mu_{\text {eff }} \equiv\left|A_{\mathrm{H}}\right| \sigma=\frac{n_{1} \mu_{\text {н1 } 1} \mu_{1}+n_{2} \mu_{\mathrm{H} 2} \mu_{2}}{n_{1} \mu_{1}+n_{2} \mu_{2}}
$$

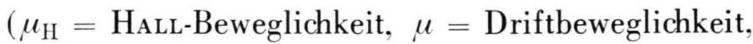
$1=$ Leitungsband, $2=$ Störband) .

Da $\mu_{\mathrm{H} 2} \ll \mu_{\mathrm{H} 1}$, gilt im Maximum von $\left|A_{\mathrm{H}}\right|$ $n_{1} \mu_{1} \approx n_{2} \mu_{2}$. Setzt man einmal $\mu \approx \mu_{\mathrm{H}}$ und und $\mu_{1} / \mu_{2}$ unabhängig von $T$, so ergibt sich für $\left|A_{\mathrm{H}}\right|{ }_{\text {max }} \sigma$ näherungsweise $\mu_{1} / 2$. Um den Wert der Beweglichkeit der Elektronen im Leitungsband $\mathrm{zu}$ erhalten, müssen also die $\left|A_{\mathrm{H}}\right| \sigma$-Meßwerte bei den Temperaturen der Maxima verdoppelt werden. Wie man der Abb. 5 entnehmen kann, passen sich die so bestimmten $\mu$-Werte ausgezeichnet an den bei Temperaturen über $10^{\circ} \mathrm{K}$ ermittelten Verlauf an, so daß der gezeichnete starke Abfall aufgehoben ist. Damit ist gezeigt, daß dieser Kurventeil ursächlich mit der Störbandleitung und mit einem Zweibändermechanismus verknüpft ist und nicht mehr als $\mathrm{H}_{\text {ALL }}$ Beweglichkeit gedeutet werden kann. Weiterhin erhält man aus dem Verhältnis $\operatorname{der} A_{\mathrm{H}}$-Werte im Maximum und im Gebiet vollständiger Ionisation der Störstellen

$$
\left|A_{\mathrm{H}}\right| \max \left(N_{\mathrm{D}}-N_{\mathrm{A}}\right) e \approx \frac{N_{\mathrm{D}}-N_{\mathrm{A}}}{4 n_{1}} \approx \frac{1}{4} \frac{\mu_{1}}{\mu_{2}} .
$$

Bei den vorliegenden Ergebnissen wird

$$
\mu_{2} \approx 0,3 \mathrm{~cm}^{2} / \text { Volt sec bei Probe } 15 \text { für } 5,5{ }^{\circ} \mathrm{K}
$$

und $\mu_{2} \approx 3,5 \mathrm{~cm}^{2} /$ Volt sec bei Probe 17 für $10,0{ }^{\circ} \mathrm{K}$. Die Unterschiede in diesen etwas hypothetischen Werten sind unter Berücksichtigung der Störstellenverhältnisse in den genannten Proben durchaus plausibel.

Unsere Messungen können also im Rahmen der geschilderten Modellvorstellung qualitativ verstanden werden, wobei in Übereinstimmung mit theoretischen Forderungen der große Einfluß der p-n-
Kompensation evident ist. Es darf noch erwähnt werden, daß auch die Messungen der magnetischen Widerstandsänderung an diesen Kristallen in qualitativem Einklang mit diesen Überlegungen sind ${ }^{24}$.

Abschließend sei noch auf ein nicht geklärtes Faktum hingewiesen. Die Leitfähigkeitskurven der Abb. 3 zeigen zwischen $6{ }^{\circ} \mathrm{K}$ und $8{ }^{\circ} \mathrm{K}$ leichte Knickpunkte, die offensichtlich durch Anomalien in den $\mu(T)$-Werten verursacht sind. Dieser eigenartige Verlauf der Beweglichkeits-Temperatur-Kurven ist von uns schon häufiger an Sb-dotierten Ge-Kristallen beobachtet worden. Mit aller Vorsicht könnte man versuchen, diesen Effekt mit Hilfe der von SCLAR ${ }^{33}$ entwickelten Theorie der Ladungsträgerstreuung an ionisierten Störstellen bei tiefen Temperaturen zu deuten. Da die übliche Borssche Näherung bei der Behandlung der Elektronenstreuung an ionisierten Zentren in diesem Gebiet nicht mehr gültig ist, braucht das bekannte $T^{3 / 2}$-Gesetz nicht erfüllt zu sein. Es läßt sich zeigen, daß bei bestimmten Potentialverhältnissen und Elektronengeschwindigkeiten ein Ramsauer-Effekt auftreten und die Beweglichkeit entsprechend verändern kann.

\section{Verhalten im Stoßionisationsbereich $\left(U \approx U_{\mathrm{D}}\right)$}

Die vorstehend beschriebenen Meßresultate werden erheblich verändert, wenn die elektrische Feldstärke sich dem kritischen Wert $U_{\mathrm{D}}$ nähert oder diesen sogar überschreitet. Hier treten Abweichungen vom OHmschen Gesetz auf, die Probenwiderstände werden feldstärkeabhängig. Abb. 6 zeigt diesen $\mathrm{Zu}$ sammenhang für die n-Ge-Kristalle 15, 17 und 18 bei $4,2{ }^{\circ} \mathrm{K}$. Die Kurven werden vollständig reversibel durchlaufen und sind bei der gleichen Probe quantitativ reproduzierbar. Offensichtlich besteht qualitativ die schon aus früheren Arbeiten 23, 26, 27 her bekannte Korrelation zwischen dem Beginn des Steilabfalles, also dem $U_{\mathrm{D}}$, und der Probenreinheit. Die sauberste Probe 15 hat die niedrigste „Durchschlagsfeldstärke", der höchste Wert wird bei dem stark p-n-kompensierten Kristall 17 gefunden.

Bei der Festlegung der kritischen Feldstärken können wir verschiedene Definitionen benutzen. Es erscheint nach der Art der Kurven der Abb. 6 wenig sinnvoll, dem $U$-Wert besondere Bedeutung beizumessen, bei dem die ersten Abweichungen vom OHmschen Gesetz auftreten. So definieren auch ScLar

${ }^{33}$ N. Sclar, Phys. Rev. 104, 1548 [1956]. 
und Burstein ${ }^{26}$ ein $U_{\mathrm{D}}$ in der Weise, daß sie das Maximum der Neigung $\Delta R / \Delta U$ bestimmen, während Koenig und Gunther-Mohr ${ }^{27}$ den Abszissenwert einer senkrechten Kurventangente in einem StromSpannungs-Diagramm als $U_{\mathrm{D}}$ wählen. In der vorliegenden Arbeit haben wir den letzten senkrechten Abfall der reversiblen $R(U)$-Kurven mit der Abszisse zum Schnitt gebracht und diese Feldstärke $U_{\mathrm{D}}$ genannt. Diese Definition stimmt im wesentlichen mit der Festlegung von Koenig und Gunther-Mohr überein. Sie führt in den Fällen zu anderen Werten, bei denen die Feldstärken in den Proben mit abnehmendem Widerstand absinken (negative Widerstände) ${ }^{24}$.

Um nachzuweisen, daß die beobachteten Effekte auf Vorgängen im Halbleiterinnern beruhen, sind verschiedene Testversuche ausgeführt worden. In Übereinstimmung mit Sclar und Burstern ${ }^{26}$ sind die Versuchsergebnisse unabhängig von der benutzten Ätzmethode, vom geometrischen Verhältnis Volumen/Oberfläche, von einer Neukontaktierung der Spannungssonden - es wurde auch hier stets mit getrennten Strom- und Spannungszuführungen gearbeitet - und von einem transversalen elektrischen Feld. Diese Befunde machen es sehr wahrscheinlich, daß die Oberflächen keinen nennenswerten Einfluß haben. Das wird auch weiterhin durch die noch zu beschreibende magnetische Einwirkung bestätigt.

Als Ursache für diese bemerkenswerte Erscheinung darf man bei normalem Störleitungsmechanismus die Ladungsträgervervielfachung durch Stoßionisation ansehen ${ }^{18}$, wobei man noch darüber diskutieren kann, welche Stellen durch den Aufprall der Elektronen ionisiert werden. Den unmittelbaren Nachweis für diese Auslegung bringen HALL-EffektMessungen, die eine Abnahme der HaLL-Konstanten ergeben, deren Betrag fast quantitativ mit der Widerstandsabnahme übereinstimmt. Damit ist gleichzeitig klar, daß sich bei dieser Art Proben auch die Trägerbeweglichkeit im Feld nicht wesentlich ändert. Leider war es uns aus apparativen Gründen (zu geringe Kapazität der He-Verflüssigung) wegen der zu großen Joule-Wärme nicht möglich, den „Durchschlag“ bis zu den Trägerdichten der Störstellenerschöpfung vorzutreiben. Impulsmessungen von Sclar und Burstein lassen aber bei diesen Konzentrationen einen stark verzögerten Abfall der $R(U)$. Kurven erkennen. Es ist daher naheliegend, von einer Stoßionisation neutraler Störstellen zu sprechen. Diese Tatsache macht auch die völlige Reversibilität des Effektes verständlich. Aus den gleichen
Impulsmessungen folgt außerdem als Zeitkonstante für das An- und Abklingen der Elektronenlawinen ein Wert unter $10^{-8} \mathrm{sec}$.

Eine einfache korpuskulare Betrachtung liefert als Abschätzung, daß etwa $10-15$ quasielastische $\mathrm{Zu}$ sammenstöße der Elektronen mit dem Gitter oder den Gitterstörungen notwendig sind, bis das stoBende Teilchen die Störstellenaktivierungsenergie erreicht. Mit einer Beweglichkeit von $2 \cdot 10^{5} \mathrm{~cm}^{2}$ pro Volt sec bei $10^{\circ} \mathrm{K}$ (Abb. 5) folgt in einem elektrischen Feld von $10 \mathrm{Volt} / \mathrm{cm}$ eine Driftgeschwindigkeit von $2 \cdot 10^{6} \mathrm{~cm} / \mathrm{sec}$. Dieser Wert liegt bereits über der thermischen Geschwindigkeit der Elektronen von etwa $1,6 \cdot 10^{6} \mathrm{~cm} / \mathrm{sec}$, so daß die Feldwirkung schon eine starke Störung der Elektronenverteilung darstellt. Die hier nicht berücksichtigte Statistik wird diese Werte noch etwas verändern, da die schnellsten Elektronen der Verteilung bei diesen Vorgängen besonders ins Gewicht fallen.

Nach diesen Überlegungen kommt der Trägerbeweglichkeit für das Zustandekommen der Stoßionisation besondere Bedeutung zu. Aus den Messungen in den Abb. $3-5$ ist aber bekannt, daß zumindest bei den Proben 15 und 17 bei $4,2{ }^{\circ} \mathrm{K}$ durch die Störbandleitung eine erhebliche Verminderung der effektiven Beweglichkeit bedingt ist. Hier müßte man also in Ergänzung zu dem beschriebenen Verhalten der Halbleiter im Bereich der Störstellenhalbleitung normaler Art annehmen, daß z. B. die in ihrer Konzentration um viele Zehnerpotenzen verminderten, aber recht schnellen Elektronen des Leitungsbandes die Stoßionisation verursachen. Die HaLL-Konstante sollte in diesem Falle infolge einer Vermehrung der Elektronen im Leitungsband auf Kosten der Elektronen im Störband wieder ansteigen. Das widerspricht den experimentellen Ergebnissen. So haben wir in einer früheren Arbeit ${ }^{23}$ Messungen der Leitfähigkeit und des HaLL-Effektes an der Probe 17 in Abhängigkeit von der elektrischen Feldstärke bei $8,5{ }^{\circ} \mathrm{K}$ und $9,5^{\circ} \mathrm{K}$ mitgeteilt. Auch bei diesen Temperaturen befindet sich der Kristall bereits im Gebiet der überwiegenden, wenn auch noch nicht vollständigen Störbandleitung. Aus (4 a) und (4 b) und den Abschätzungen für das Verhältnis $\mu_{1} / \mu_{2}$ folgt, daß hier zwar $n_{1} \mu_{1} \ll n_{2} \mu_{2}$, aber noch $n_{1} \mu_{1}^{2} \gg n_{2} \mu_{2}^{2}$ gilt. Dann ergibt sich

$$
\begin{gathered}
A_{\mathrm{H}}=\frac{1}{e} \frac{n_{1} \mu_{\mathrm{H} 1} \mu_{1}}{n_{2}{ }^{2} \mu_{2}{ }^{2}}, \\
\mu_{\mathrm{eff}}=\frac{n_{1} \mu_{\mathrm{H} 1} \mu_{1}}{n_{2} \mu_{2}} .
\end{gathered}
$$


Die in der Abb. 2 der zitierten Arbeit ${ }^{23}$ wiedergegebene Verminderung von $\left|A_{\mathrm{H}}\right|$ mit steigendem $U \gtrsim U_{\mathrm{D}}$ ist mit einer Zunahme von $n_{1} / n_{2}$ nicht verträglich. Die aus den Meßwerten ermittelte effektive Beweglichkeit $\left|A_{\mathrm{H}}\right| \sigma_{\mathrm{H}}$ ist in der Abb. 7 als Funktion der elektrischen Feldstärke dargestellt. Nach einem nahezu konstanten Verlauf erfolgt im Abfallgebiet des Widerstandes eine Beweglichkeitsverminderung um mehr als eine Größenordnung, im Gegensatz zu den Messungen an Proben außerhalb der Störbandleitung (z. B. 18) und im Gegensatz zu einer $\mathrm{Zu}$ nahme von $n_{1} / n_{2}$ nach Gl. (6 b) .

Es bliebe allerdings noch der Ausweg, eine extrem starke Verminderung der Trägerbeweglichkeit $\mu_{1}$ zu postulieren und dann trotz einer Zunahme von $n_{1} / n_{2}$ noch eine Abnahme von $\left|A_{\mathrm{H}}\right|$ zu erzielen. Da es sich hier um Größenordnungsveränderungen handelt, müßte diese Herabsetzung von $\mu_{1}$ auch mehrere Zehnerpotenzen umfassen. Das ist aber nach den Daten der Tab. 1 für diese Probe sehr unwahrscheinlich. Wohl tritt mit zunehmender Störstellenionisation eine Verminderung von $\mu_{1}$ infolge einer $\mathrm{Zu}$ nahme der Zahl von ionisierten Zentren ein, letztere kann aber höchstens von $2 N_{\mathrm{A}}$ auf $N_{\mathrm{D}}+N_{\mathrm{A}}$, also bei Probe 17 nur um einen Faktor 1,03 wachsen. Die Beweglichkeit selbst wird etwas stärker variieren, da nach SCLAR ${ }^{33}$ und BLATT ${ }^{34}$ die anziehenden Zentren einen größeren Wirkungsquerschnitt aufweisen als die abstoßenden. Eine größenordnungsmäßige Änderung von $\mu_{1}$ ist jedenfalls nicht plausibel.

Eine Deutungsmöglichkeit hat Sсноттку angegeben ${ }^{35}$. Bei dem Fortwandern eines Elektrons im Störband wird es bei seinem Eintreffen an einer Nachbarstörstelle eine - noch nicht erklärte Gitterrelaxation hervorrufen und unter Austausch von Schwingungsenergie mit dem Gitter gebremst. Das Fortwandern nimmt daher den Charakter einer Diffusion mit Vorzugsrichtung an. Die Existenz einer endlichen Aktivierungsenergie ist durch die Messungen von FrITzSCHE $^{6}$ bewiesen. Sie liegt bei Sb-dotierten Ge-Kristallen bei 1,6 $10^{-3} \mathrm{eVolt}$. Wird dieser Energiebetrag dem Teilchen bei einem Sprung durch das äußere elektrische Feld zugeführt oder ist die aufgenommene Energie gar größer, so vermag das Elektron an der Nachbarstörstelle vorbeizuwandern. Eine Bremsung in stärkeren elektrischen Feldern wird nunmehr von den thermischen Gitterschwingungen übernommen. Das Teilchen kann als quasifrei und der durch das elektrische Feld ver- ursachte Vorgang als eine Art Ionisation im Störband aufgefaßt werden.

In unserem Bandmodell entspricht dieser Mechanismus einer Beweglichkeitserhöhung aller Elektronen des Störbandes. Es ist durchaus plausibel, daß sie mit einer bestimmten Feldstärke einsetzt und erhebliche Beträge annimmt. Bei konstantem $n_{1} / n_{2}$ ist damit eine Abnahme von $\left|A_{\mathrm{H}}\right|$ ebenso wie eine $\mathrm{Ab}$ -

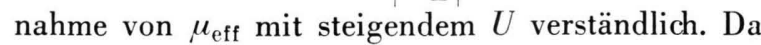
$\mu_{2}$ in $A_{\mathrm{H}}$ quadratisch, in $\mu_{\text {eff }}$ nur linear enthalten ist, sollten die Änderungen dieser Größen in einem entsprechenden Verhältnis stehen. Diese Forderung ist quantitativ nicht ganz erfüllt. Zum Beispiel fällt $\left|A_{\mathrm{H}}\right|$ von dem Wert bei $4,1 \mathrm{Volt} / \mathrm{cm}$ auf $1 / 94$ bei $10,2 \mathrm{Volt} / \mathrm{cm}$, demgegenüber nimmt $\mu_{\text {eff }}$ unter den gleichen Bedingungen auf $1 / 21$ ab. Als Ursache für diese Diskrepanz kann eine gleichzeitig auftretende, hier aber unberücksichtigte Verminderung von $\mu_{1}$ wirksam sein. Eine solche geringe Abnahme erscheint nicht so ganz unvernünftig, wenn man beachtet, daß durch die Befreiung der Teilchen des Störbandes auch alle bei kleinen elektrischen Feldern noch neutralen Störstellen zu ionisierten Zentren werden, deren Streuquerschnitt für die Elektronen des Leitungsbandes bis 10-mal größer sein kann als der der ionisierten Akzeptoren und zudem ist der von ScLar postulierte Ramsauer-Effekt bei der Elektronenstreuung außerordentlich empfindlich von kleinen Veränderungen der Dichte der ionisierten Zentren abhängig. So läßt sich eine quantitative Übereinstimmung erzielen, wenn man für $\mu_{1}$ zwischen $4,1 \mathrm{Volt} / \mathrm{cm}$ und $10,2 \mathrm{Volt} / \mathrm{cm}$ eine Verminderung um den Faktor 2,1 annimmt. $\mu_{2}$ variiert dann noch um den Faktor 4,6 .

Nach diesen Ausführungen scheint der von Sсноттку vorgeschlagene Mechanismus die Meßergebnisse im Gebiet der Störbandleitung wenigstens qualitativ erklären zu können. Es bliebe noch nachzutragen, daß man bei Störstellendichten von $10^{14} \mathrm{~cm}^{-3}$ aus dem mittleren Störstellenabstand von etwa $5 \cdot 10^{-4} \mathrm{~cm}$ schon mit Feldstärken der Größenordnung $5 \mathrm{Volt} / \mathrm{cm}$ die erforderlichen Werte der Relaxationsenergien erreichen kann. Es erscheint uns zudem denkbar, daß der bei normaler Störstellenhalbleitung experimentell bewiesene Stoßionisationsmechanismus ausgeschaltet wird, sobald die Teilchen des Störbandes quasifrei wandern können

${ }^{34}$ F. J. B Batt, J. Phys. Chem. Solids 1, 262 [1957].

35 Briefliche Mitteilung vom 26.2.1958. Die Verff. möchten Herrn Prof. Sсноттку für diesen Hinweis herzlich danken. 


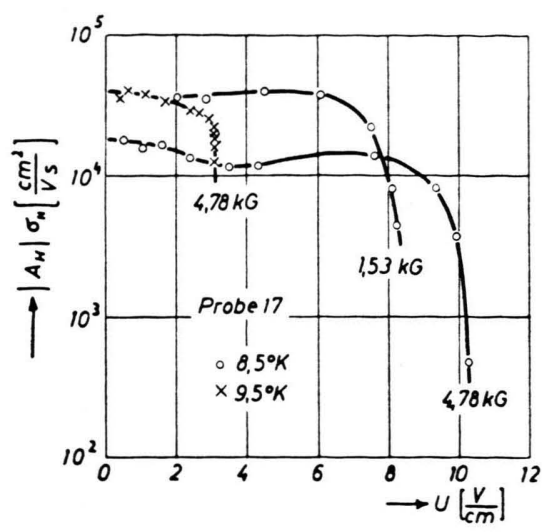

Abb. 7. Feldstärkeabhängigkeit der Größe $\left|A_{\mathrm{H}}\right| \sigma_{\mathrm{H}}$ eines p-n-kompensierten n-Ge-Einkristalls im Bereich der Störbandleitung. Parameter: Magnetfeldstärke.

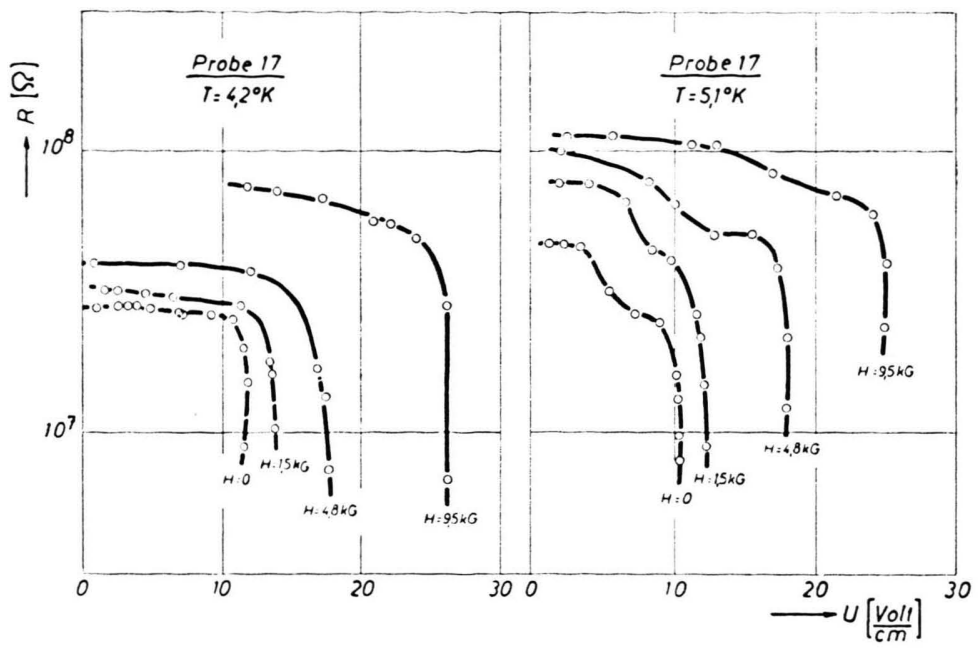

Abb. 8. Änderung der Feldstärkeabhängigkeit des Widerstandes eines n-Ge-Einkristalles mit der Temperatur. Parameter: Magnetfeldstärke. und damit eine durch Stoß behebbare Bindung an ein bestimmtes Zentrum nicht mehr vorliegt.

Unsere Interpretation der Feldstärkeabhängigkeit des elektrischen Widerstandes der Ge-Kristalle hat gewisse Konsequenzen für die Temperaturabhängigkeit der $U_{\mathrm{D}}$-Werte. In dem gewöhnlichen Störstellengebiet ist der reziproke Zusammenhang zwischen $U_{\mathrm{D}}$ und der Trägerbeweglichkeit im Leitungsband gut erfüllt ${ }^{23,26}$. Die kritischen Feldstärken nehmen daher bei allen Proben mit abnehmender Temperatur zunächst ab. Bei $T>10^{\circ} \mathrm{K}$ werden die Messungen durch Wärmeeffekte infolge der Joule-Wärme gestört, so daß quantitative Angaben fehlerhaft sind. Sicher liegen die $U_{\mathrm{D}}$-Werte bei $14,0^{\circ} \mathrm{K}$ über $25 \mathrm{Volt} / \mathrm{cm}$ im Vergleich zu $1-2 \mathrm{Volt} / \mathrm{cm}$ in der Umgebung von $10^{\circ} \mathrm{K}$. Unterhalb $10^{\circ} \mathrm{K}$ muß erwartet werden, daß sich bei den Proben 15 und 17 das Einsetzen der Störbandleitung mit dem anderen Leitungsmechanismus für $U \approx U_{\mathrm{D}}$ im Temperaturverlauf bemerkbar macht. Wie der Abb. 3 unserer früheren Arbeit ${ }^{23} \mathrm{zu}$ entnehmen ist, steigt $U_{\mathrm{D}}$ bei Probe 17 dicht unter $10^{\circ} \mathrm{K}$ auf etwa 7 Volt/cm an, um zu tieferen Temperaturen hin wesentlich schwächer zu wachsen. Diese Abhängigkeit kann mit der Trägerbeweglichkeit im Leitungsband quantitativ nicht in Einklang gebracht werden. Ein ähnlicher Anstieg ist bei der Probe 15 unter $5{ }^{\circ} \mathrm{K}$ angedeutet, also wieder in einem Bereich, in dem die Störbandleitung zu überwiegen beginnt. Die leichte Temperaturabhängigkeit der $U_{\mathrm{D}}$-Werte unterhalb dieser Über- gangstemperaturen wird durch die Begünstigung der Trägerbefreiung im Störband durch die thermische Energie verständlich ${ }^{35}$.

Eine quantitative Deutung der Experimente scheitert zur Zeit an einer Reihe ungeklärter Sekundäreffekte. So beobachtet man vielfach, daß sich die Form der $R(U)$-Kurven mit der Temperatur ändert, wie in der Abb. 8 als Beispiel dargestellt ist. Ob hier eine Überlagerung verschiedener Mechanismen der gleichen Art vorliegt, kann noch nicht definitiv entschieden werden. Offensichtlich haben auch die Kontakte unter Umständen einen Einfluß auf die Kurvenform, wenn auch der reversible $U_{\mathrm{D}}$-Wert weniger empfindlich zu sein scheint. So konnten wir z. B. bei der Probe 18 auch irreversible Änderungen mit Bereichen negativer Widerstände beobachten, die wohl mit der Trägerinjektion in nicht einwandfreien Kontakten in Zusammenhang stehen. Diese Effekte sind durch Neukontaktierung leicht zu beheben. Es darf noch erwähnt werden, daß eine Deutung des ganzen $R(U)$-Verlaufs durch avalanche injection von den Kontakten her ${ }^{36}$ wegen der geringen Feldstärken am Gesamtkristall sicher auszuschließen ist.

\section{Magnetische Beeinflussung der „Durchschlags- vorgänge ${ }^{66}$}

Aus den Abb. 7 und 8 ist ersichtlich, daß ein transversales Magnetfeld die feldstärkeabhängige 36 J. B. Gunv, Proc. Phys. Soc., Lond. B 69, 781 [1956]. 
Widerstandsabnahme verringert und die kritischen elektrischen Feldstärken $U_{\mathrm{D}}$ nach höheren Werten zu verschiebt. Diese Beobachtung findet im Rahmen der entwickelten Modellvorstellungen ihre einfache Deutung. Im Magnetfeld werden die Elektronenbahnen zwischen zwei Stoßprozessen gekrümmt. Da die mittlere freie Weglänge der Träger sicher nicht gröBer wird, nimmt der freie Flugweg in Richtung der elektrischen Feldstärke ab. Damit verringert sich die Energieaufnahme aus diesem Feld. Um die gleichen energetischen Verhältnisse wie im Falle $H=0$ zu schaffen, müssen die $U$-Werte erhöht werden. Die-

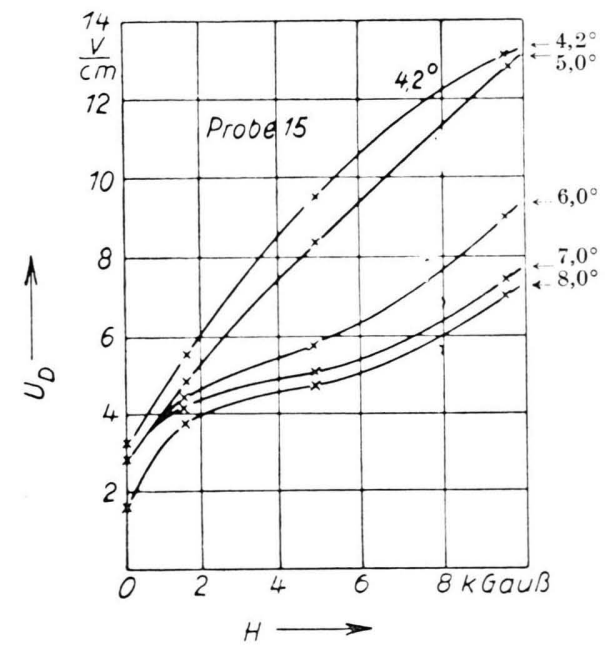

Abb. 9. Magnetfeldabhängigkeit der Durchschlagsfeldstärke eines bei Zimmertemperatur eigenleitenden n-Ge-Kristalls. Parameter: Temperatur.

ser Effekt muß sich um so stärker auswirken, je gröBer die freie Weglänge, je reiner also die Kristalle sind.

In den Abb. 9 - 11 sind die „Durchschlagsfeldstärken " $U_{\mathrm{D}}$ bei verschiedenen konstanten Temperaturen in Abhängigkeit vom Magnetfeld für die untersuchten Proben aufgetragen worden. Bei der experimentellen Bestimmung dieser Werte wurden die elektrischen und magnetischen Felder stets unabhängig voneinander umgepolt, um den evtl. Einfluß von magnetischen Sperrschichten und Raumladungseffekten an den Elektroden zu kontrollieren. In keinem Fall ist eine größere Korrektur notwendig gewesen.

Qualitativ läßt sich feststellen, daß beim Übergang zur Störbandleitung die Kurven die Tendenz zeigen, geradlinig zu werden (Probe 17, Probe 15 unter $\left.5^{\circ} \mathrm{K}\right)$, während im normalen Störleitungs- gebiet kein einheitlicher Verlauf zu finden ist. Hierbei müssen wir erwähnen, daß in diesem Bereich auch die magnetische Widerstandsänderung implizit

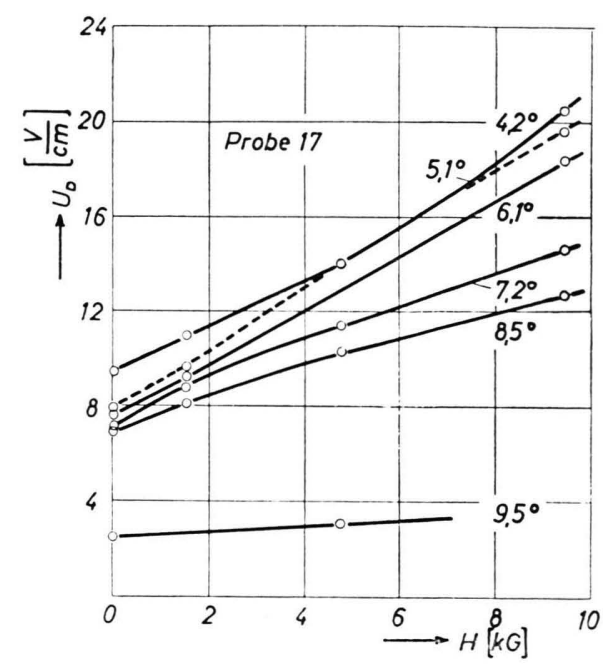

Abb. 10. Magnetfeldabhängigkeit der Durchschlagsfeldstärke eines mit In und Sb p-n-kompensierten n-Ge-Kristalls. Parameter: Temperatur.

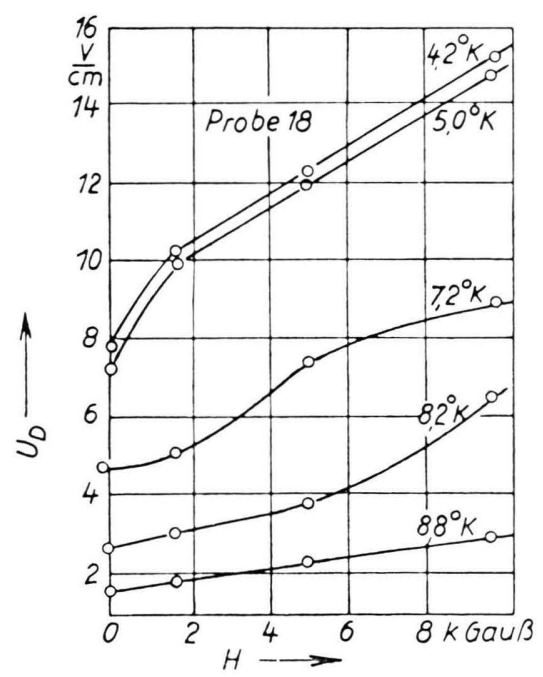

Abb. 1l. Magnetfeldabhängigkeit der Durchschlagsfeldstärke eines schwach mit Sb dotierten n-Ge-Einkristalls. Parameter: Temperatur.

eingeht, die ihrerseits nach den Untersuchungen von Lautz und Ruppel ${ }^{37}$ bei tiefen Temperaturen starke individuelle Unterschiede zwischen den Einzelproben erkennen läßt. In ursächlichem Zusammenhang da-

37 G. Lautz u. W. Ruppel, Z. Naturforschg. 10 a, 521 [1955]. 


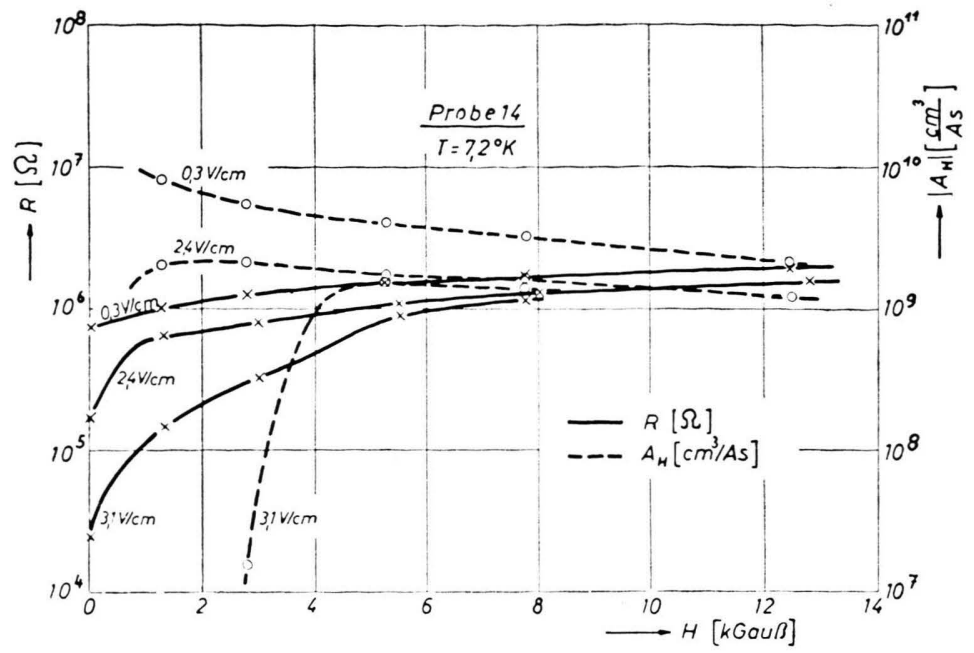

Abb. 12. Magnetfeldabhängigkeit des elektrischen Widerstandes und der HalL-Konstanten eines $\mathrm{n}-\mathrm{Ge}$-Einkristalls bei $7,2{ }^{\circ} \mathrm{K}$. Parameter: Elektrische Feldstärke. mit steht auch die mit steigendem Magnetfeld ablesbare Variation der Temperaturabhängigkeit der Feldstärken $U_{\mathrm{D}}$. Die erwartete Korrelation zwischen Probenreinheit und Anstieg der $U_{\mathrm{D}}(H)$-Kurven wird durch das Experiment bestätigt.

Ein unmittelbarer Nachweis für die Aufhebung der Stoßionisation bei normaler Störstellenhalbleitung wird durch gleichzeitige Widerstands- und HaLl-Effekt-Messungen bei verschiedenen konstanten elektrischen Feldstärken in Abhängigkeit vom Magnetfeld gegeben (Abb. 12). Bei 0,3 Volt $/ \mathrm{cm}$ liegt die normale magnetische Widerstandsänderung vor, bei $2,4 \mathrm{Volt} / \mathrm{cm}$ sind für $H=0$ schon beträchtliche Abweichungen vom OHмschen Verhalten beobachtbar, bei 3,1 Volt/cm ist der $U_{\mathrm{D}^{-}}$Wert fast erreicht. Die Widerstandskurven nähern sich mit steigendem Magnetfeld einander an, ohne sich jedoch völlig zu überdecken. Zwischen 5000 und $6000 \mathrm{Gauß}$ ist die Widerstandsverminderung durch Stoßionisation weitgehend behoben. Der Sachverhalt wird auch durch die in der Abb. 12 eingetragenen $A_{\mathrm{H}}(H)$-Werte gut bestätigt. Die auch bei kleinen elektrischen Feldern noch vorhandene Abnahme der HaLL-Konstanten kann bei dieser Messung durch Randeinflüsse infolge ungünstiger Probendimensionierung bedingt sein. Entsprechend dem oft viel komplexeren Verhalten einzelner Proben (vgl. Abb. 8) ist auch bei dem Magnetfeldeinfluß unter Umständen eine stufenweise Aufhebung der erhöhten Leitwerte aufzufinden.

38 W. FrIE, Dissertation, Münster 1958; Z. Naturforschg. 14 a, 54 [1959], voranstehend.
Durch die vorstehenden Betrachtungen finden die großen relativen magnetischen Winderstandsänderungen der Proben ohne Störbandleitung $\left(\Delta \varrho / \varrho_{0} \approx 10000 \%\right.$ bei $300 \mathrm{Gau} \beta$ und $\left.4,2{ }^{\circ} \mathrm{K}\right)$ und deren Abhängigkeit von der elektrischen Feldstärke eine einfache Erklärung. Diese Ergebnisse wurden schon in unserer früheren Arbeit ${ }^{23}$ diskutiert. In Übereinstimmung mit den Experimenten wird die Zunahme der $\Delta \varrho / \varrho_{0}$-Werte als Funktion von $H$ bei jenen elektrischen Feldstärken maximal, bei denen der Beginn des Steilabfalles der $R(U)$-Kurven einsetzt. Für $U \ll U_{\mathrm{D}}$ bleibt nur die vergleichsweise kleine, normale magnetische Widerstandsänderung, für $U>U_{\mathrm{D}}$ sind zur Beeinflussung der Stoßionisation größere $H$-Werte erforderlich.

Die hier mitgeteilten Untersuchungen haben FrIE zu theoretischen Betrachtungen über den Einfluß des Magnetfeldes auf die Stoßionisation angeregt ${ }^{38}$. In Fortführung und Erweiterung der Theorie von FrANZ $^{39}$ findet FrIE, daß die Magnetfeldeinwirkung in der Energiebilanz durch die Einführung einer mit den veränderten Stoßwahrscheinlichkeiten zusammenhängenden effektiven Feldstärke statt der normalen elektrischen Feldstärke für $H=0$ beschrieben werden kann. Für diese Größe ergibt sich bei beliebigen magnetischen Feldstärken ein recht komplexer Ausdruck. Zudem sind die Ergebnisse von der kristallographischen Orientierung abhängig. Im Falle starker Magnetfelder und tiefer Temperaturen

39 W. Franz, Handbuch der Physik XVII, Springer-Verlag, Berlin 1956, S. 155. 
besteht ein linearer Zusammenhang mit $H$, sofern das Magnetfeld in der Richtung [100] oder [111] liegt. Für ein Magnetfeld in Richtung [110] erhält man einen Sättigungswert. Wie FrIE zeigen kann, ergibt sich mit Hilfe seiner Theorie aus den linearen Kurven der Abb. 9 (Probe 15) ein durchaus vernünftiger Wert für die Stoßzahl, so daß eine befriedigende Übereinstimmung besteht.
Abschließend möchten wir Herrn Prof. Justi für die Möglichkeit zur experimentellen Durchführung dieser Arbeit herzlich danken. Besonderer Dank gilt den Herren Prof. Sснотткy und Prof. Franz für wertvolle Hinweise und Diskussionen. Die Einkristalle wurden uns dankenswerterweise von Herrn F. W. Deнmelt (Telefunken) zur Verfügung gestellt. Die Deutsche For $\mathrm{schungsgemeinschaft} \mathrm{hat} \mathrm{die} \mathrm{Untersuchungen}$ durch Sachbeihilfen und Leihgaben großzügig unterstützt.

\title{
Theorie der Kleinwinkelstreuung von Röntgen-Strahlen und Neutronen durch innere Spannungen in festen Körpern, insbesondere durch Versetzungen
}

\author{
Von Alfred Seeger und Ekkehart Kröner \\ Aus dem Institut für theoretische und angewandte Physik der Technischen Hochschule Stuttgart \\ und dem Max-Planck-Institut für Metallforschung, Stuttgart \\ (Z. Naturforschg. 14 a, $74-80$ [1959]; eingegangen am 3. September 1958)
}

\begin{abstract}
Die von Versetzungen herrührende Kleinwinkelstreuung, die bisher als Integral über das Dilatatationsfeld der Versetzungen (und anderer Fehlstellen) ermittelt werden mußte, wird (soweit sie sich nach der linearen Elastizitätstheorie behandeln läßt) als Integral über die Versetzungsdichte dargestellt. Für Versetzungslinien (sog. singuläre Versetzungen) reduziert sich dieses Integral auf ein entlang der Versetzungslinien zu erstreckendes Linienintegral, mit dessen Hilfe die Rechnungen über die Kleinwinkelstreuung von Versetzungen sehr vereinfacht werden. Als Beispiel wird die Kleinwinkelstreuung durch kreisförmige Stufenversetzungsringe behandelt. Solche Ringe können durch die Kondensation von Leerstellen in Kristallen entstehen und spielen neuerdings eine Rolle bei der Diskussion der Vorgänge in abgeschreckten Metallen.
\end{abstract}

Für die Streuung von Röntgen-Strahlen ist die Verteilung der Elektronen maßgebend. Betrachtet man einen Kristall einheitlicher chemischer Zusammensetzung, insbesondere einen Elementkristall (z. B. ein Metall), so ist bei genügend großer Ordnungszahl die Elektronendichte praktisch proportional der Dichte der Materie, da man unter diesen Bedingungen den Einfluß der Valenzelektronen neben demjenigen der Rumpfelektronen vernachlässigen kann. Durch die Kleinwinkelstreuung von RöNTGENStrahlen werden bekanntlich die Variationen in der Elektronenverteilung, die durch die atomare Struktur der Kristalle bedingt sind, nicht erfaßt, wohl aber diejenigen Dichtevariationen, die sich über eine größere Zahl von Atomabständen hinweg erstrekken. Für die Beschreibung solcher Dichtevariationen eignet sich naturgemäß die Elastizitätstheorie besonders gut, so daß man zur Behandlung der Kleinwinkelstreuung durch innere Spannungen in festen Körpern zweckmäßigerweise von der im Sinne der Kontinuumsgeometrie definierten Volumdilatation $\Theta(\mathfrak{r})$ als Funktion des Ortsvektors $\mathfrak{r}$ ausgeht. Man kann zeigen ${ }^{1}$, daß die Amplitude der Kleinwinkel- streuung näherungsweise durch die dreidimensionale Fourier-Transformierte der Dilatation, also durch den Ausdruck

$$
A(\mathfrak{g})=\iiint \Theta(\mathfrak{r}) \exp (-i \mathfrak{g} \cdot \mathfrak{r}) \mathrm{d} \tau_{\mathrm{r}},
$$

erstreckt über den ganzen Kristall, bestimmt ist. Hierbei ist der sog. Beugungsvektor $\mathfrak{g}$ als vektorielle Differenz zwischen dem Wellenvektor $\mathfrak{f}^{\prime}$ der gestreuten und dem Wellenvektor $\mathfrak{f}$ der einfallenden Welle, also durch

$$
\mathfrak{g}=\mathfrak{f}^{\prime}-\mathfrak{f},
$$

definiert. Bezeichnet $2 \vartheta$ den sog. Streuwinkel, d. h. den Winkel zwischen $\mathfrak{f}^{\prime}$ und $\mathfrak{f}$, so hängt der Betrag $g$ des Beugungsvektors mit der Wellenlänge $\lambda$ gemäß

$$
g=\frac{4 \pi \sin \vartheta}{\lambda}
$$

zusammen ${ }^{2}$.

1 Siehe hierzu z. B. A. Guinier u. G. Fournet, Small-Angle Scattering of X-Rays, John Wiley \& Sons, Inc., New York 1955 sowie A. SEeger, International Conference on the Small-Angle Scattering from Metals, Kansas City 1958 (Veröffentl. J. appl. Phys., demnächst).

2 Diese Bezeichnungen sind dieselben, wie sie früher verwendet wurden (A.SEEGER,Z.Naturforschg.11 a,724 [1956]). 